\title{
Synthesis of Data on the Local Interste1lar Medium
}

Donald G. York* and Priscilla C. Frisch

Department of Astronomy and Astrophysics

University of Chicago

\section{Introduction}

During this workshop, a considerable amount of data was presented, some refined versions of earlier results and some entirely new. While no completely definite picture of the local interstellar medium can be presented yet, some general conclusions can be drawn. We attempt to draw together the most relevant data in Section III. Some new UV results are collated with published results to help state the physical properties of the gas. A morphological view of the local medium is given in Section III. We confine ourselves to the region within 100 pc with the caveat that the distance of much of the material under discussion is poorly known.

\section{Data on the Local Interstellar Medium}

\section{A) Neutral Hydrogen $\left(\mathrm{T}<10^{4}{ }^{\circ} \mathrm{K}\right)$}

Two views of the distribution of neutral hydrogen near the Sun can be derived from observations. First, large scale features in $21 \mathrm{~cm}$ all sky maps show high latitude loops and extensions above the galactic plane (Cleary et a1. 1979). These loops form one or two "she11s" roughly centered on the sight-line to the Sco-Oph association and known as "Loop I" or the "Sco-Oph Bubble" (Berkhuijsen et al. 1971, Weaver 1979). From $21 \mathrm{~cm}$ line widths, the shel1 gas material has $\mathrm{T}<400^{\circ} \mathrm{K}$ (Heiles 1982) and it is observed when $\mathrm{N}(\mathrm{HI})>$ $5 \times 10^{19} \mathrm{~cm}^{-2}$. Studies of NaI absorption at the known velocity of specific filaments show that much of the material in Loop I is within $100 \mathrm{pc}$ of the Sun (Frisch and York, this volume). Polarization studies (Mathewson and Ford 1970) confirm that interstellar dust with polarization vectors aligned along the Loop I she1l is evident in stars from $50-100$ pc from the Sun. This dust also shows up directly on $100 \mu$ IRAS maps as elongated dust structures (or "cirrus") along the shell. The observations of nearby dust by Knude (this volume) and Perry and Johnson (1982), as well as nearby small Co clouds (Blitz this volume), may also sample some of this shell material. The magnetic field threading the shell has $B \sim 6 \mu G$ (Heiles et al. 1980, Vallee preprint).

A second view based on UV observations sensitive to neutral gas with $\mathrm{N}(\mathrm{HI}) \geqslant 10^{17} \mathrm{~cm}^{-2}$ was formulated by Frisch and York 1983. Figure 1 shows a view of a $500 \mathrm{pc}$ region looking down on the plane. Figure 2 shows a 100 pc region looking at the Sun from $1=270^{\circ}, b=0^{\circ}$. Gas within the shaded contours is generally cool $\left(\mathrm{T}<200^{\circ} \mathrm{K}\right)$, from observations of molecular hydrogen and its rotational excitation structure (Savage et al. 1977), Spitzer and Morton

*Also a member of the Enrico Fermi Institute 


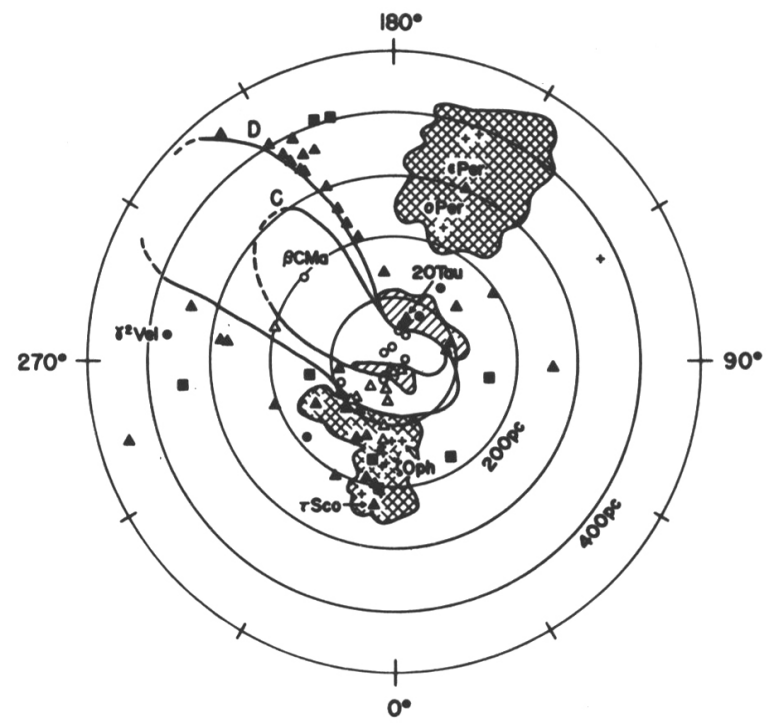

Figure 1: Nearby neutral hydrogen distribution as viewed looking down on the galactic plane. Contours A, B, C and D correspond to $\mathrm{N}(\mathrm{H})=0.05,0.25,0.5$ and $5 \times 10^{19} \mathrm{~cm}^{-2}$, respectively. See Frisch and York (1983) for details.

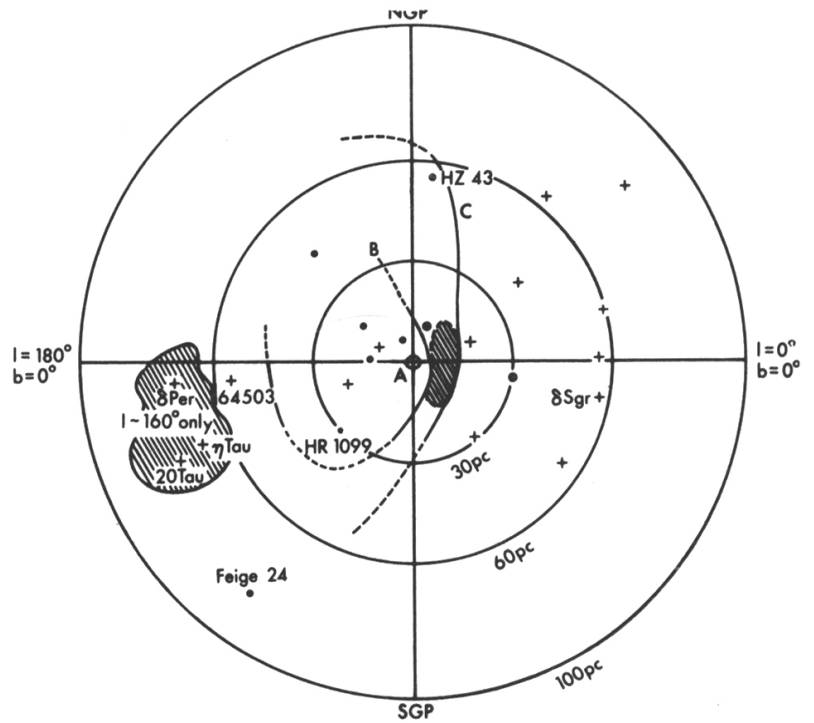

Figure 2: Same as Figure 1, but viewed from $1=270^{\circ}, b=0^{\circ}$. 
1976), observations of $21 \mathrm{~cm}$ line widths (Crutcher, this volume), and observations of the hyperfine splitting of $\mathrm{NaI} D$ lines (Blades et al. 1980).

A warm component of HI is, however, evident in the UV data. Table 1 gives b-values for neutral species in the main neutral velocity components toward four stars, all of which lie in quadrants III $\left(180^{\circ}\right.$ to $\left.270^{\circ}\right)$ and IV $\left(270^{\circ}\right.$ to $\left.360^{\circ}\right)$ in Figure 1 . The components have $\mathrm{N}(\mathrm{HI})<3 \times 10^{19} \mathrm{~cm}^{-2}$, and, according to the apparent decrease of line width with nuclear mass $\mathrm{T}>6000^{\circ} \mathrm{K}$. The small cloud near the center of Figure 1 may cover a large enough solid angle to explain these warm components toward $\alpha \mathrm{Vir}, \lambda \mathrm{Sco}$, and $\beta$ Cen (a11 at distances $\dot{i} 100 \mathrm{pc}$ ). The Ly $\alpha$ and HeI $\lambda 584$ backscattered solar radiation discussed by several authors in this volume may also be related to this feature. Table 2 gives some constraints on the size of the HI regions toward the four stars used in Table 1 .

Crutcher (1982) summarizes the local NaI observations that show a preferential direction of motion for gas seen in nearby stars. He concludes all the data are consistent with a $\mathrm{V}_{\mathrm{HC}}=-28 \mathrm{~km} \mathrm{~s}^{-1}$ flow from the "apparent" upwind direction $1=25^{\circ}, \mathrm{b}=+10^{\circ}$, corresponding to $V_{L S R}=-15 \mathrm{~km} \mathrm{~s}^{-1}$ from the "true" (or LSR) upwind direction $1=345^{\circ}, b=-10^{\circ}$. Table 3 gives, for stars within $100 \mathrm{pc}$ (or further for low column density paths), the velocity data for UV components where $V_{H I}$ is the velocity of the dominant HI component and $V_{H I I}$ is the velocity of the dominant HII component. Additional components are readily discerned from profiles of strong UV lines (York 1983). These are, in general of mixed ionization, with $\mathrm{N}(\mathrm{HI}+\mathrm{HII})$ between $10^{16} \mathrm{~cm}^{-2}$ and $10^{18} \mathrm{~cm}^{-2}$. The number of such components and the minimum and maximum velocity found in each direction are also given. It is apparent that there is a tendency for all gas components to have more negative velocities in the direction toward the galactic center, more positive velocities away from it. On the other hand the values $V_{H I}$ suggest more negative velocities than either Crutcher's NaI analysis or the Ly $\alpha$ backscatter of solar radiation from neutral interstellar material within the solar system (e.g., Adams and Frisch 1977, Weller and Meier 1981, and several papers in this volume) although several star positions are very close to the true upwind direction. We conclude that there is a general flow of nearby material, including regions with different physical properties, originating from a direction near the center of Loop I.

Much more information is potentially available on the details of motions of neutral gas near the Sun using MgI and MgII observations (Bruhweiler et al., Beckman et a1., de Boer and Lenhart, all in this volume, Vidal Madjar private communication). However, $\mathrm{MgII}$ is formed in both neutral and ionized gas and the necessary detailed breakdown into HI/HII components has not yet been done for these data.

\section{B) Ionized Gas $\left(T<10^{5}{ }^{\circ} \mathrm{K}\right)$}

Observations of nearby stars often show regions with SII and other ions in absorption, but are accompanied by neutral nitrogen, argon, and oxygen with $N(X I) / N(S I I)<\sim 0.1(N(X) / N(S))$, where $X$ is nitrogen, argon, or oxygen and $\mathrm{N}$ is the column density in units of $\mathrm{cm}^{-2}$. These are apparently HII regions. 
Table 1: b Values in Neutral Components

$\begin{array}{lcccc} & \alpha \mathrm{Vir}^{1} & \lambda \mathrm{Sco}^{2} & \beta \mathrm{Cen}^{3} & \beta \mathrm{CMa}^{4} \\ \text { HI } & 11(+1,-1) & 11(+1,-1) & 12(+1,-1) & 14.0(+0.5,-0.5) \\ \text { DI } & 6(+2,-2) & 8(+3,-2) & 8(+2,-2) & 9-12 \\ \text { OI } & 4(+0.5,-1.0) & 2.4(+.1,-0.1) & 2(+1,-1) & 6-8: \\ \text { NI } & 4.7(+0.3,-0.2) & 2.5(+.1,-.1) & 3(+1,-1) & 3-7 \\ \text { ArI } & 2.7(+0.3,-0.2) & 1.7(+0.2,-0.2) & 3(+0.5,-1.0) & ---\end{array}$

${ }^{1}$ York and Kinahan 1979; ${ }^{2}$ York 1983; ${ }^{3}$ York, in preparation; ${ }^{4} \mathrm{Gry}$, V1dal-Madjar, and York in preparation.

Table 2: HI Regions Near the Sun

\begin{tabular}{|c|c|c|}
\hline Star & Size & Method \\
\hline$\alpha$ Vir ${ }^{1}$ & $<5 \mathrm{pc}$ & $\begin{array}{l}\text { Some } C I I^{*} \text { in HI since HII region observed }{ }^{2} \text { is } \\
\text { larger than CII*/CII implies }\end{array}$ \\
\hline$\lambda \mathrm{Sco}$ & $<5 \mathrm{pc}$ & CII* at $\mathrm{HI}$ vel. \\
\hline B CMa & $<5 \mathrm{pc}$ & Same $N(H I)$ to $\alpha \mathrm{CMa} 0.3 \mathrm{pc}$ away from line of sight \\
\hline B Cen & $<10 \mathrm{pc}$ & CII* seen at HI vel. \\
\hline
\end{tabular}

Table 3: Velocities of Dominant Interstellar Components in Nearby Stars (LSR velocities given)

\begin{tabular}{|c|c|c|c|c|c|c|c|c|c|}
\hline \multirow{2}{*}{ Star } & \multirow[b]{2}{*}{1} & \multirow[b]{2}{*}{ b } & \multicolumn{3}{|c|}{ (dom) (dom) } & \multirow[b]{2}{*}{ No. $>10^{16}$} & \multirow[b]{2}{*}{$\mathrm{V}_{\mathrm{min}}$} & \multirow[b]{2}{*}{$\mathrm{V}_{\max }$} & \multirow[b]{2}{*}{$\mathrm{N}(\mathrm{OVI})^{7}$} \\
\hline & & & d & $\mathrm{V}_{\mathrm{HI}}$ & $\mathrm{V}_{\mathrm{HII}}$ & & & & \\
\hline$\eta \mathrm{UMa}$ & 100 & 65 & 29 & --- & --- & -- & --- & -- & $<12.4$ \\
\hline - And ${ }^{2}$ & 102 & -16 & 90 & 0 & -16 & 6 & -16 & +24 & -- \\
\hline$\delta \operatorname{Per}^{3}$ & 150 & -6 & 82 & -5 & +4 & 6 & -9 & +27 & $<13.7$ \\
\hline $20 \mathrm{Tau}^{2}$ & 166 & -24 & 78 & 1,9 & $-\infty$ & 5 & +1 & +27 & --- \\
\hline$n \mathrm{Tau}^{2}$ & 167 & -23 & 59 & 8,16 & -1 & 4 & -1 & +16 & --- \\
\hline$\alpha$ Leo & 226 & 49 & 22 & -- & --- & -- & --- & $--\dot{-}$ & $<13.4$ \\
\hline$\beta \mathrm{CMa}^{4}$ & 226 & -14 & 214 & $(-1)$ & +9 & 6 & -10 & +26 & $<11.4$ \\
\hline$\alpha \operatorname{Er} 1^{2}$ & 291 & -59 & 22 & 0 & -- & 5 & -15 & +17 & 13.1 \\
\hline$\beta \mathrm{Cen}^{3}$ & 312 & 1 & 100 & -13 & -5 & 6 & -30 & +12 & 13.0 \\
\hline$\alpha \operatorname{V} 1 r^{1}$ & 316 & 51 & 87 & -7 & -15 & 4 & -23 & +2 & 13.4 \\
\hline$\alpha \mathrm{Gru}^{2}, 4$ & 350 & -52 & 29 & -9 & -- & 3 & -17 & -1 & $<12.9$ \\
\hline$\lambda \mathrm{Sco}^{5}$ & 352 & -2 & 100 & -32 & -23 & $5(+1 ?)$ & -40 & -8 & 13.3 \\
\hline u Sco 6 & 351 & -2 & 134 & -32 & -22 & 4 & -39 & -12 & $-\infty$ \\
\hline
\end{tabular}

${ }^{1}$ Same reference as Table 1; ${ }^{2}$ Martin (1981); ${ }^{3}$ Martin and York (1982); ${ }^{4}$ York (unpublished); ${ }^{5}$ York (1983); ${ }^{6}$ Eder (1983); ${ }^{7}$ Jenkins (1978). 
Observations of excited CII from these regions allows derivation of electron densities. Doppler broadening of the lines from these regions may occur, but $\mathrm{T}_{\mathrm{HII}}<12000{ }^{\circ} \mathrm{K}$ in all cases except $\beta \mathrm{CMa}$, where $\mathrm{T}_{\mathrm{HII}}<10^{5}{ }^{\circ} \mathrm{K}$ from considerations of b-values alone. Table 4 lists known HII regions from UV absorption data which are or may be near the Sun (the case of $\beta$ CMa is ambiguous).

In several lines of sight, SiIII and CII absorption lines require the presence of material at velocities not seen in other species ( $\alpha$ Vir, $\lambda \mathrm{Sco}$ ). From considerations of ionization equilibrium, such gas has $\mathrm{T}>50000^{\circ} \mathrm{K}$, and it consists only of low column densities, $\mathrm{N}(\mathrm{HI}+\mathrm{HII})<10^{17} \mathrm{~cm}^{-2}$.

From all sky studies of $\mathrm{H} \alpha$ emission maps Reynolds (this volume) concludes that $\mathrm{H} \alpha$ emission often comes from regions with radii>10 pc and $\mathrm{n}_{\mathrm{e}}>0.1$. A particular region 1ikely to be near the Sun is centered on $\alpha \operatorname{Vir}$ at $d=100$ pc.

Table 4: HII Regions Near the Sun ${ }^{1}$

\begin{tabular}{|c|c|c|c|c|}
\hline Star & $\mathrm{CII*} / \mathrm{CII}^{2}$ & $\mathrm{n}_{\mathrm{e}}$ & $2 r$ & Near Star? \\
\hline$\alpha \operatorname{Vir}$ & $<0.04$ & $<0.5$ & $>6 \mathrm{pc}$ & Yes $(H \alpha)$ \\
\hline$\lambda \mathrm{Sco}$ & 0.006 & 0.2 & $30 \mathrm{pc}$ & Yes(Eders 1982) \\
\hline B $\mathrm{CMa}$ & 0.003 & 0.1 & $60 \mathrm{pc}$ & $?$ \\
\hline$\beta$ Cen & 0.1 & 1 & $6 \mathrm{pc}$ & $?$ \\
\hline
\end{tabular}

${ }^{1}$ References as for Table 1.; ${ }^{2}$ The entry is $\mathrm{N}(\mathrm{CII} *) / \mathrm{N}(\mathrm{CII})=\mathrm{N}(\mathrm{CII} *) /[\mathrm{N}(\mathrm{CII}) *$ $\left.\left.(\mathrm{C} / \mathrm{S})\right|_{0}\right]$

Studies of MgII with Copernicus and IUE (several papers this volume) trace HI and HII gas indiscriminately. Every derivation from N(MgII) of $N(H)=N(H I)+N(H I I)$ in any given direction requires an assumption about the abundance of magnesium in interstellar gas. Magnesium may have a solar abundance (Dufton et a.1. 1984) or less (de Boer et a1. this volume). However for all nearby stars studied so far in $\mathrm{MgII}, \mathrm{N}(\mathrm{H})<10^{20} \mathrm{~cm}^{-2}$ and $\mathrm{N}(\mathrm{HII})<10^{20} \mathrm{~cm}^{-2}$, in particular. Observations, in fewer stars (Table 4), of SII indicate $\mathrm{N}(\mathrm{HII})<3 \times 10^{19} \mathrm{~cm}^{-2}$ within $100 \mathrm{pc}$ of the Sun. It is noteworthy that $\mathrm{N}(\mathrm{HII})>\mathrm{N}(\mathrm{HI})$ toward $\left(190^{\circ}, 0^{\circ}\right)$ over a $200 \mathrm{pc}$ path length to $\beta \mathrm{CMa}$.

\section{C) Hot Gas $\left(\mathrm{T}>10^{5}{ }^{\circ} \mathrm{K}\right)$}

Jenkins (this volume) reviews the fact that observations of absorption Iines of OVI are consistent with the existence of gas within $100 \mathrm{pc}$ with $\mathrm{T} \sim 4 \times 10^{5}{ }^{\circ} \mathrm{K}$. Theoretically, the ratio $\mathrm{N}(\mathrm{OVI}) / \mathrm{N}\left(0_{\text {total }}\right)$ drops dramatically with either an increase or decrease of temperature, so the absence of indications of gas at hotter or cooler temperatures in OVI is probably misleading. Some material at $10^{5}{ }^{\circ} \mathrm{K}$ may be present, but $\mathrm{CIV}$ and $\mathrm{NV}$, the prime indicators of $10^{5}{ }^{\circ} \mathrm{K}$ gas, are weak (corresponding to $\mathrm{N}(\mathrm{HII})<10^{17.5}$ at $10^{5}{ }^{\circ} \mathrm{K}$ ) and may come entirely from the OVI producing regions. Small pockets of such gas may exist near white dwarfs within $100 \mathrm{pc}$ (Raymond this volume). 
$\mathrm{X}$-ray emission over the entire sky has been observed near $78 \AA$ (M-band), $56 \AA$ (C-band), and $16 \AA$ (B-band) (various papers in this volume by McCammon et a1., Sanders et al., Clark). It is argued that at $b<60^{\circ}$, the observed emission in the low energy $B$-band must be local because dark shadows from clouds are not seen toward any direction. The HI regions outside $100 \mathrm{pc}$ (FIgure 1) have $\mathrm{N}(\mathrm{HI})>5 \times 10^{19} \mathrm{~cm}^{-2}$ and would certainly absorb X-rays from beyond. Since cloud shadows are not seen, the emission must be foreground to the HI. In the higher energy M-band, the region interfor to Loop I and the North Polar Spur are bright, with a spectral softening towards the edge of the emitting region caused by the Loop I HI shell (Iwan 1980).

For $b>60^{\circ}$, large regions of enhanced $X$-ray emissions are seen. In this case, a strong anticorrelation of HI emission and X-ray emission is seen (Clark, this volume) but the expected decrease in the ratio of $B$ band $X$-rays to $C$ band $X$-rays as HI emission increases is not seen (McCammon this volume). Several authors (this volume) concluded that very small high column density H I clouds not delineated in broad beam $21 \mathrm{~cm}$ surveys could offer a solution to this dilemma, since they would be opaque to both of the two lowest energy bands yet allow some radiation to come through unattenuated. On the other hand, small scale $21 \mathrm{~cm}$ structure that would allow this is ruled out based on several studies (this volume).

\section{Synthesis}

The above data can be fitted together in the following way, as plctured in Figure 3. A three dimensional model of local stars and interstellar gas has been constructed treating $\mathrm{H} I$ associated with Loop $I$ and the more distant Sco-Oph filaments as a single giant bubble of radius roughly $90 \mathrm{pc}$. This model was photographed from a position corresponding to $1=180^{\circ}$. The cotton represents HI from the Heiles maps and the dust clouds around the Plelades (left foreground). In this model, the Sun is located in the shell of the bubble. The tight clump of stars at center left represents the Sun, $\alpha$ Cen $\mathrm{AB}$, and $\alpha \mathrm{CMaAB}$. White dwarfs are small, stars hotter than $\mathrm{F}$ are blue, cooler stars are yellow (in reference to the color slide used at the conference - copies may be obtained from the authors). Ionized gas seen in CII* with Copernicus and/or in $\mathrm{H} \alpha$ emission (Reynolds this volume) fills large sections of the empty space (perhaps $10-30 \%$ of the volume). The large holes at $1=230^{\circ}$ and $1=100^{\circ}$ are probably filled by HII regions seen toward $\gamma$ Cas (Ferlet et al. 1980) and toward B CMa (Gry et al., this volume).

The remainder of the void must be filled by the X-ray emitting gas. The brightening at the poles may be caused by shocks induced by blast waves from high latitude supernova (Pop I) that travel unimpeded through low density hot gas above the disk, but are slowed and emit X-rays on encountering HI gas in the plane. If higher density clouds lead to greater slowing and softer $\mathrm{X}$-ray emission, the detalls of the $\mathrm{X}$-ray flux discussed above may find a natural explanation.

Both interpretations of the soft $\mathrm{X}$-ray data and theoretical discussions at this meeting have indicated the possibility of a supernova event possibly 
$10^{5}$ years ago that reheated an already hot cavity (Iwan 1980, Cowie this volume, Edgar and Cox this volume, Rocchia et al. this volume). The models place the X-ray emission primarily in an 1dealized shell of HI, so the sky is uniform at $b<60^{\circ}$ in $X$-ray emission even if the Sun is off center. The high velocity CII and SIII components (Table 1) may be a consequence of the blast wave.

Detalled observation of OVI profiles for stars at $d<100 \mathrm{pc}$ are available (see Table 3). OVI may come from interfaces between the hot X-ray emitting gas and the clouds (HI or HII) at $\mathrm{T}<10^{5}{ }^{\circ} \mathrm{K}$ (Cowie et al. 1979). These 11nes are narrow and well defined in $\alpha$ Vir, but broad in $\lambda$ Sco and $\beta$ Cen. The stars $\gamma$ Cas and $\beta$ CMa show no OVI to very high limits. Considerations of projected velocities of the nearby cloud of warm HI (Figure 2) and the HII region velocities towards nearby stars (Table 3) offer a potential explanation of these results. Toward $\lambda$ Sco and $\beta$ Cen we may be seeing evaporation from the far side of the nearby cloud and from the near side of HII regions around these stars. Evaporation from the warm cloud near the Sun toward $1 \sim 0^{\circ}$ at a higher velocity than the HII regions could cause the broadening of OVI profiles toward these two stars. Toward $\alpha$ Vir, the HI and HII regions have (accidentally) simflar velocities, so evaporation from interfaces with both could produce profiles of nominal thermal width because they coincide closely in velocity space. (See York 1977, Cowie et al., 1979 for detalls of the data and the evaporation possibility). The absence of OVI towards the stars $\gamma$ Cas (Jenkins 1978) and $\beta$ CMa (Table 3), where both $\mathrm{T}<20000^{\circ} \mathrm{K}$ clouds and $X$-ray emission are seen, is yet to be explained. The many different types of gas and their distribution in space would seem to indicate that the relatively smooth $B$-band X-ray emission around the Sun masks a rather complex irregular distribution of X-ray emitting gas.

The warm HI toward $\alpha$ Vir and $\lambda$ Sco may well be within $20 \mathrm{pc}$ of the sun (Ferlet et al. this volume). The various evidences of gas near the sun indicate there may be a single close cloud containing warm HI with a minimum extension of $25 \mathrm{pc}$ parallel to the plane of the sky. If this cloud is a fragment of the Loop I shell it is probably much larger and may have a magnetic field of $B \sim 6 \mu \mathrm{G}$ threading it. Interstellar polarization is seen in stars in this direction as close as $5 \mathrm{pc}$ (Tinbergen this volume). The Ly $\alpha$ backscatter suggests parts of this cloud are within a few AU of the Sun. So $f a r$, we have no good indication of what lies in the wake of the Sun if it is actually moving through this cloud. This cloud has mean neutral density $\mathrm{n}_{\mathrm{H}}<5 \mathrm{~cm}^{-3}$, but backscattering data implies a lower density, $\mathrm{n}_{\mathrm{H}^{\sim}} 0.1 \mathrm{~cm}^{-3}$, near the Sun. Refractory trace elements in this cloud show relatively high abundances indicating it may have been shocked (Frisch 1981, York 1983). The similar column densities of HI toward $\alpha \mathrm{CMa}$ and $\beta \mathrm{CMa}$, in spite of the great distance to the latter, suggest an extension of the warm cloud into Quadrant III consistent with the view that the Sun is in the shell of the Loop I bubble. 


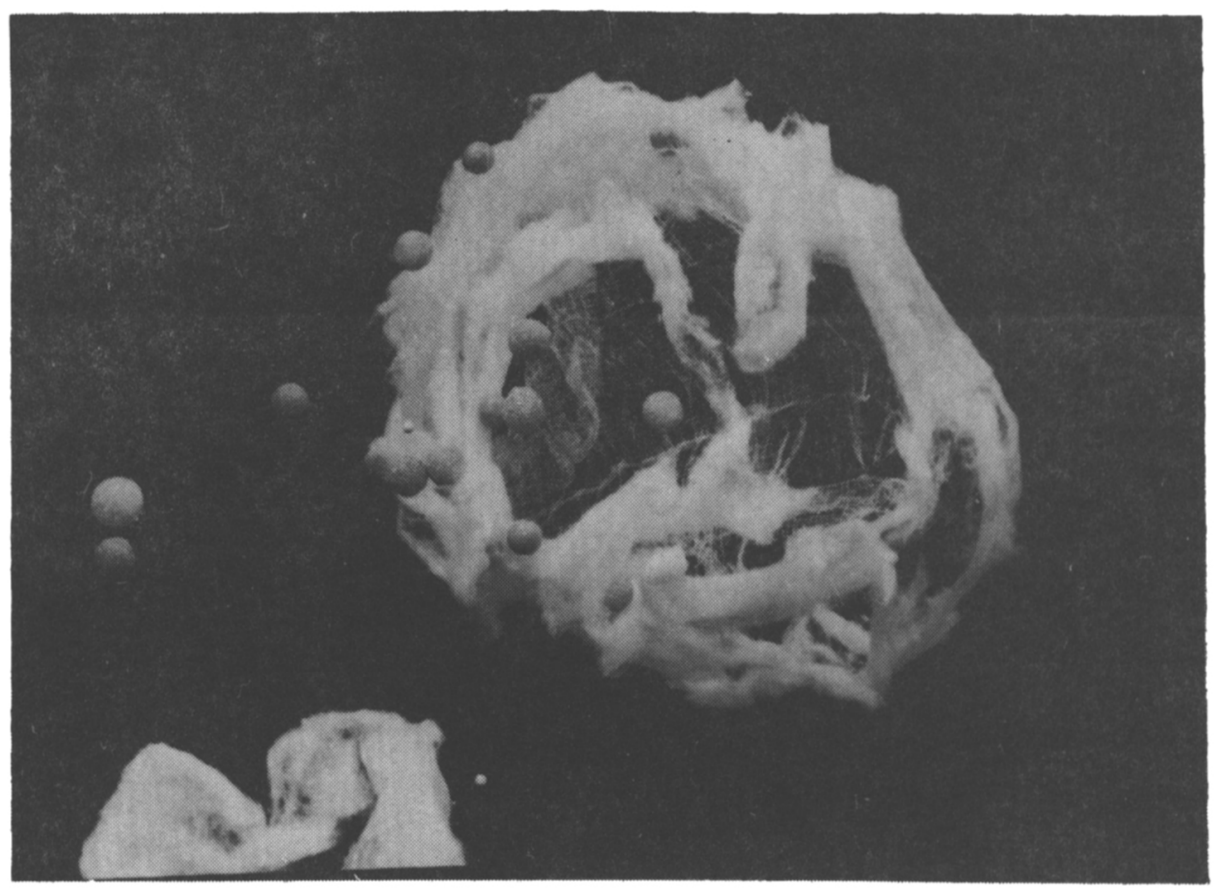

Figure 3: Three dimensional model of nearby interstellar gas with Loop I and the Sco-Oph bubble treated as a single giant bubble. Picture taken from the position corresponding to $1=180^{\circ}$ and $b=0^{\circ}$.

\section{References}

Adams, T.F., and Frisch, P.C. 1977, Ap. J., 212, 300.

Berkhuijsen, E.M., Haslem, C.G.T., and Salter, C.J. 1971, Astr. Ap., 14, 252. Blades, J.C., Wynne-Jones, I., and Wayte, R.C. 1980, M.N.R.A.S., 193, 849 . Bohlin, R.C., Hill, J.K., Jenkins, E.B., Savage, B. D., Snow, T.P., Spitzer, L. and York, D.G. 1983, Ap. J. Supp1. Ser., 51, 277.

Cleary, M.N., Heiles, C., and Haslam, C.G.T. 1979, Astr. Ap. Suppl. Ser., $36,95$.

Cowie, L.L., Jenkins, E.B., Songaila, A., and York, D.G. 1979, Ap. J., 232, 467.

Crovisier, J., Kazes, I., and Aubry, D. 1978, Astr. Ap. Suppl. Ser., 32, 205. Crutcher, R.M. 1982, Ap. J., 254, 82.

Dufton, P.L., Hibbert, A., Murray, M.J., and York, D.G. 1984, Ap. J., to be published.

Eders, D.C. 1983, Ph.D. Thesis, Princeton University Observatory.

Ferlet, R., Vidal-Madjar, A., Laurent, C., and York, D.G. 1980, Ap. J., $242,576$.

Frisch, P.C. 1981, Nature, 293, 377.

Frisch, P.C., and York, D.G. 1983, Ap. J. (Letters), 271, L59.

Heiles, C. 1982, Ap. J., 262, 135.

Heiles, C., Chu, Y.H., Reynolds, R.J., Yegingil, I., and Troland, T.H. 1980, Ap. J., 242, 533. 
Iwan, D. 1980, Ap. J., 239, 316.

Jenkins, E.B. 1978, Ap. J., 219, 845.

Martin, E.R. 1981, Ph.D. Thesis, Princeton University Observatory.

Martin, E.R., and York, D.G. 1982, Ap. J., 257, 135.

Mathewson, D.S., and Ford, V.L. 1970, Mem. R. Astr. Soc., 74, 139.

Perry, C.L., Johnston, L. and Crawford, P.L. 1982, Ap. J., Supp1. Ser., 50, 451.

Savage, B.D., Bohlin, R.C., Drake, J.F., and Budich, W. 1977, Ap. J., 216, 291.

Spitzer, L., and Morton, W.A. 1976, Ap. J., 204, 731.

Weaver, H. 1979, Proc. IAU Symp. No. 84, ed. W.B. Burton (Dordrecht \& Reide1), 295.

Weller, C.is., and Meier, R.R. 1981, Ap. J., 246, 386.

York, D.G. 1977, Ap. J., 213, 43.

York, D.G. 1983, Ap. J., 264, 172 .

York, D.G., and Kinahan, B.F. 1979, Ap. J., 228, 127. 